\title{
The mechanism of domain-wall structure formation in Ar-Kr submonolayer films on graphite
}

\author{
A. Patrykiejew, S. Sokołowski \\ Department for the Modelling of Physico-Chemical Processes, Faculty of Chemistry, MCS University, 20031 \\ Lublin, Poland
}

Received July 18, 2014, in final form October 23, 2014

\begin{abstract}
Using Monte Carlo simulation method in the canonical ensemble, we have studied the commensurateincommensurate transition in two-dimensional finite mixed clusters of $\mathrm{Ar}$ and $\mathrm{Kr}$ adsorbed on graphite basal plane at low temperatures. It has been demonstrated that the transition occurs when the argon concentration exceeds the value needed to cover the peripheries of the cluster. The incommensurate phase exhibits a similar domain-wall structure as observed in pure krypton films at the densities exceeding the density of a perfect $(\sqrt{3} \times \sqrt{3}) R 30^{\circ}$ commensurate phase, but the size of commensurate domains does not change much with the cluster size. When the argon concentration increases, the composition of domain walls changes while the commensurate domains are made of pure krypton. We have constructed a simple one-dimensional FrenkelKontorova-like model that yields the results being in a good qualitative agreement with the Monte Carlo results obtained for two-dimensional systems.
\end{abstract}

Key words: adsorption of mixtures, commensurate-incommensurate transitions, Monte Carlo simulation, finite systems, Frenkel-Kontorova model

PACS: $68.35 . R h, 64.70 . R h, 64.60 . a n$

\section{Introduction}

The commensurate-incommensurate (C-IC) transitions in adsorbed films have been experimentally observed in a variety of systems [1-15] and studied by theoretical methods [16-27] and computer simulations [28-37].

In a large number of systems, the incommensurate phase forms a collection of finite commensurate regions (domains) separated by domain walls [21]. The domain wall networks appear when the $C$ phase is degenerated, i.e., when the atoms (or molecules) in the $C$ phase occupy one of several equivalent lattices. The stability of IC phases with domain walls networks is considerably enhanced by large entropic effects [16, 21]. The most prominent example of such a system is the krypton monolayer adsorbed on the graphite basal plane [38-41]. At low temperatures, the adsorption of krypton leads to the formation of the three-fold degenerate commensurate $(\sqrt{3} \times \sqrt{3}) R 30^{\circ}(\sqrt{3} \times \sqrt{3}) R 30^{\circ}$ structure, in which every krypton atom occupies one of every three hexagons of the graphite surface, has the lattice constant $\sqrt{3}$ times the graphite lattice constant ( $a_{\mathrm{gr}}=2.46 \AA$ ) and is rotated with respect to the graphite lattice by 30 degrees. Upon the increase of pressure, the commensurate phase undergoes a transition into the incommensurate phase of higher density, in which the commensurate domains are separated by walls, and the atoms in neighboring domains are shifted to a different sublattice [40, 42-44]. In this case, the walls are called heavy and carry all the excess of density. At the densities just above the C-IC transition the domains are very large and their size gradually decreases when the density increases towards the monolayer completion. This has been clearly demonstrated by a large scale computer simulation study [30].

The C-IC transition has also been experimentally observed in the mixed Kr-Xe and Ar-Xe films on graphite [45-48]. On the other hand, the structure of mixed Ar-Kr films on graphite has not been experimentally studied yet. The only available experimental results were published by Singleton and Halsey 
nearly 60 years ago [49] and demonstrated that mixed liquid-like films exhibit complete mixing, independently of the composition. The only information about the structure and properties of solid-like mixed $\mathrm{Ar}-\mathrm{Kr}$ films on graphite have been obtained using computer simulation methods [50, 51]. In particular, it has been demonstrated that already at submonolayer coverages and at sufficiently low temperatures, the addition of argon into krypton film triggers the C-IC transition, and leads to the formation of finite clusters in which small commensurate domains consisting of pure krypton are separated by heavy walls built of argon and krypton [51]. The C-IC transition in one-component finite clusters of Lennard-Jones atoms adsorbed on graphite has also been studied by Houlrik et al. [37]. They have used Monte Carlo method to study the effects of the corrugation potential on the structure of finite systems and have shown that for a given amplitude of the corrugation potential the presence of free surfaces enhanced the stability of the $\mathrm{C}$ structure.

In this paper we study the mechanism of the C-IC transition of submonolayer mixed films made of argon and krypton using Monte Carlo simulation methods and appropriately modified one-dimensional (1D) Frenkel-Kontorova (FK) model [52, 53]. The original FK model handles an infinite chain of atoms interacting via harmonic potential and subjected to a periodic (sinusoidal) external field at zero temperature. The FK model can be used to describe the basis features of C-IC transitions [17]. At this point, we should mention that the FK model has been extended to two-dimensional systems [54, 55] to mixtures [56], systems with disorder [57, 58] and has also been used to study finite chains [59-63].

In one of our recent works [52], we studied the impurity driven commensurate-incommensurate transitions in one-dimensional finite systems using the FK model. We assumed that the pure chain is commensurate and discussed the effects of impurities located either only at one end or at two ends of the chain. It is shown that in both situations the C-IC transition occurs when the amplitude of the external field experienced by the impurity atoms falls into the region between the lower and upper threshold values. These limiting values of the amplitude depend upon the parameters characterizing the interaction between the atoms in the main chain, the amplitude of external field acting on the main chain atoms and the interaction between the main chain and the impurities. The number of solitons (domain walls) in the IC structure were found to depend upon the chain length, and the parameters describing the interactions in the system. Our findings have been confirmed by Monte Carlo simulation in two-dimensional systems.

Here, we also considered finite chains of $\mathrm{Kr}$ atoms with impurities (Ar atoms) located at the chain ends as well as in the chain interior.

The paper is organized as follows. In the next section we presented the two-dimensional model and described the Monte Carlo method used. The results of Monte Carlo simulation are presented in section 3. Then, in section 4 we presented the modified 1D FK model and demonstrated that it yields the results qualitatively very similar to those emerging from the two-dimensional Monte Carlo simulation.

\section{The two-dimensional model and simulation method}

We have considered strictly two-dimensional mixed submonolayer films consisting of $\mathrm{Ar}$ and $\mathrm{Kr}$ atoms. The interaction between the atoms is modelled via the truncated $(12,6)$ Lennard-Jones potential

$$
u_{i j}(r)= \begin{cases}4 \varepsilon_{i j}\left[\left(\sigma_{i j} / r\right)^{12}-\left(\sigma_{i j} / r\right)^{6}\right], & r \leqslant r_{\max }, \\ 0, & r>r_{\max },\end{cases}
$$

where $r$ is the distance between a pair of atoms, the subscripts $i$ and $j$ mark the species Ar and $\mathrm{Kr}$, and the potential is cut at the distance $r_{\max }=3.0 \sigma_{i j}$. The potential parameters for a pair of unlike atoms are given by the Lorentz-Berthelot relations:

$$
\sigma_{\mathrm{ArKr}}=\frac{1}{2}\left(\sigma_{\mathrm{Ar}}+\sigma_{\mathrm{Kr}}\right) \quad \text { and } \quad \varepsilon_{\mathrm{ArKr}}=\sqrt{\varepsilon_{\mathrm{Ar}} \varepsilon_{\mathrm{Kr}}} .
$$

The external field due to the graphite substrate is assumed to be given by [33, 51]

$$
v(x, y)=-V_{\mathrm{b}, i}\left\{\cos \left(\mathbf{q}_{1} \mathbf{r}\right)+\cos \left(\mathbf{q}_{2} \mathbf{r}\right)+\cos \left[\left(\mathbf{q}_{1}-\mathbf{q}_{2}\right) \mathbf{r}\right]\right\},
$$


Table 1. Lennard-Jones parameters for Ar and $\mathrm{Kr}$ used in this work

\begin{tabular}{|c|c|c|}
\hline \hline$i, j$ & $\sigma_{i, j}, \AA$ & $\varepsilon_{i, j}, \mathrm{~K}$ \\
\hline $\mathrm{Ar}, \mathrm{Ar}$ & 3.4 & 120.0 \\
$\mathrm{Kr}, \mathrm{Kr}$ & 3.6 & 171.0 \\
$\mathrm{Ar}, \mathrm{Kr}$ & 3.5 & 142.83 \\
\hline \hline
\end{tabular}

where $\mathbf{q}_{1}$ and $\mathbf{q}_{2}$ are the reciprocal lattice vectors of the graphite basal plane and the amplitudes $V_{\mathrm{b}, i}(i=$ $\mathrm{Ar}, \mathrm{Kr}$ ) determine the potential barriers between adjacent minima. We assumed here that $V_{\mathrm{b}, \mathrm{Ar}}^{*}=8.4 \mathrm{~K}$ and $V_{\mathrm{b}, \mathrm{Kr}}^{*}=14.4 \mathrm{~K}[51]$.

The values of parameters entering the potential (2.1) are given in table 1 . The graphite lattice constant $a_{\mathrm{gr}}=2.46 \AA$ is assumed to be a unit of length and $\varepsilon_{\mathrm{Ar}}$ is taken as a unit of energy.

The Monte Carlo simulation is carried out in the canonical ensemble [64] for systems of different (submonolayer) density, of different total number of atoms, $N$, and of different mole fraction of argon, $x_{\mathrm{Ar}}$, between 0 and 0.55 . We used a standard Metropolis sampling and two types of moves are taken into account, i.e., the translation of a randomly chosen atom by a randomly chosen vector within the circle of radius $d_{\max }$ and the identity exchange. The magnitude of $d_{\max }$ is updated every 1000 Monte Carlo steps (each Monte Carlo step consists of $N$ attempts to move a single atom as well as $N$ attempts to change the identity of randomly chosen atoms) in order to keep the acceptance rate equal to about 0.3 . The equilibration and production runs consist of $5 \cdot 10^{6}$ and $5 \cdot 10^{7}$ Monte Carlo steps, respectively. Standard periodic boundary conditions were used therein.

\section{The results of Monte Carlo study of Ar-Kr submonolayer films}

Since our study was performed for finite clusters at low temperatures it seems reasonable to begin with the estimation of the effects of free boundaries on the ground state properties of finite commensurate patches made of pure krypton.

We performed Monte Carlo calculations at low temperatures using different starting configurations. At first, the rectangular simulation cells of the size $L_{x} \times L_{y}=L \times L \sqrt{3} / 2$ with $L=30,60$ and 120, were used. The number of $\mathrm{Kr}$ atoms in a perfect and fully covered $(\sqrt{3} \times \sqrt{3}) R 30^{\circ}$ commensurate structure was equal to $N_{\mathrm{Kr}}=L^{2} / 3$. Then, we modified the simulation cell by making $L_{x}$ larger $\left(L_{x}=L+\Delta\right)$, while keeping the number of $\mathrm{Kr}$ atoms unchanged, i.e., equal to $N_{\mathrm{Kr}}=L^{2} / 3$. In this way, two free boundaries running along the $y$-axis were created. Of course, the distance between these two boundaries, given by $\Delta$, should be larger than the cutoff distance of the Lennard-Jones potential [equation [2.1]). The second series of calculations were done for finite hexagonal clusters of the commensurate phase built of a different number of krypton atoms. The simulation was carried out at reduced temperatures between 0.01 and 0.1 and the energies obtained were extrapolated to $T=0$. In this way, we estimated the ground state energies.

In the case of a fully occupied rectangular box, we did not observe any finite size effects, and the ground state energy (per particle) of the commensurate phase was estimated to be equal to $e_{0, \mathrm{c}}=-4.617$ (see figure 1). Similar calculations carried out for the systems with free boundaries along the $y$-axis led to the ground state energy equal to $e_{0, \mathrm{f}}=-4.562$ (see figure 1) for three different systems of the size $(60 \times 15 \sqrt{3})\left(N_{\mathrm{Kr}}=600\right),(60 \times 45 \sqrt{3})\left(N_{\mathrm{Kr}}=1800\right)$ and $(90 \times 30 \sqrt{3})\left(N_{\mathrm{Kr}}=1200\right)$.

In the ground state, the excess interfacial energy per unit length due to the presence of free boundaries of the total length $L_{\text {int }}$ can be calculated as follows:

$$
E_{\text {int }}=\left(E_{0, \mathrm{f}}-E_{0, \mathrm{c}}\right) / L_{\mathrm{int}},
$$

where $E_{0, \mathrm{f}}=N_{\mathrm{Kr}} e_{0, \mathrm{f}}$ and $E_{0, \mathrm{c}}=N_{\mathrm{Kr}} e_{0, \mathrm{c}}$ are the total energies of the systems with and without free boundaries. For the three systems mentioned above, we obtained the same value of the excess interfacial energy at $T=0$ equal to about 0.635 . This indicates that finite size effects are negligibly small at very low temperatures. 


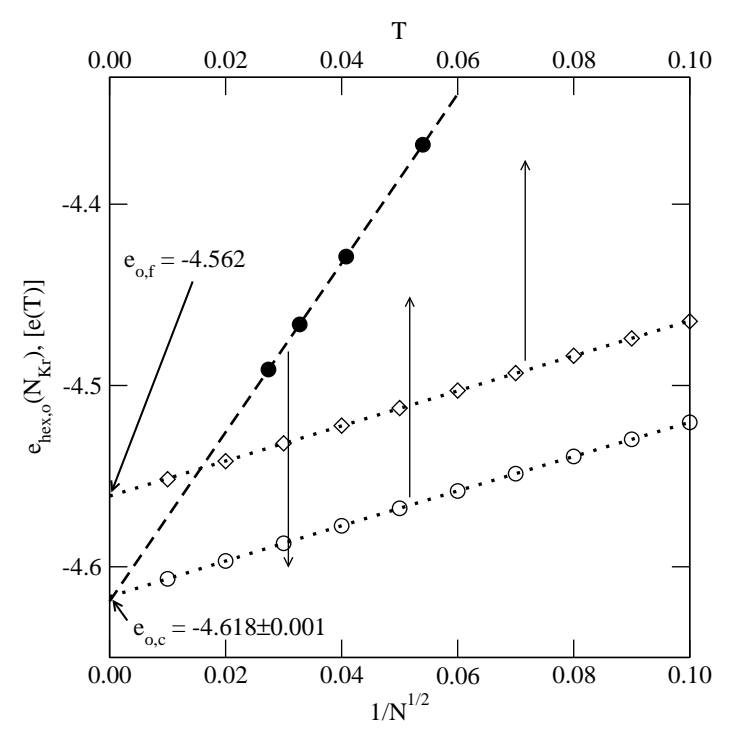

Figure 1. The temperature changes of the energy (per particle) of fully covered (open circles) and with free boundaries (open diamonds) commensurate phase obtained using a rectangular slabs containing 600, 1200 and 1800 atoms and of different total length of the free interface, $L_{\text {int }}=L_{y} \sqrt{3}$, with $L_{y}=30,60$ and 90 . The filled circles are the ground state energies of finite hexagonal patches plotted against $N^{-1 / 2}$.

On the other hand, the ground state energies of finite hexagonal clusters are bound to show large finite size effects. In such clusters, the fraction of atoms at the patch boundaries is proportional to $1 / \sqrt{N}$. In order to extrapolate the results to the limit $N_{\mathrm{Kr}} \rightarrow \infty$, we made the plot (given in figure1) of the ground state energies (per atom) for the clusters of different size $e_{\mathrm{hex}, 0}\left(N_{\mathrm{Kr}}\right)$ against $1 / \sqrt{N}$ and extrapolated the results to $1 / \sqrt{N}=0$. In this limit, the boundary effects should vanish and the energy for $N_{\mathrm{Kr}} \rightarrow \infty$ should be equal to $e_{0, \mathrm{c}}$. The obtained value is equal to -4.169 and agrees very well with the value of $e_{0, \mathrm{c}}$ obtained for the fully covered surface. The above presented results demonstrate that our simulation method gives reliable results.

It was demonstrated in our earlier work [51], that finite submonolayer clusters made of the mixture of $\mathrm{Ar}$ and $\mathrm{Kr}$ atoms exhibit domain-wall structures, in which the commensurate domains made of krypton are separated by the domain walls of varying composition. Here, we considered such systems in a more systematic way, aiming at the determination of conditions necessary for the domain-wall structure to appear.

The Monte Carlo simulations were carried out for a series of hexagonal clusters of a different total number of atoms equal to 343, 601, 931 and 1333 and of also different argon mole fraction between 0.08 and 0.55 . The calculations were performed over a rather wide range of reduced temperatures between 0.01 and 0.45 . Figure 2 shows the temperature changes of the potential energy (per particle) in the systems of different cluster size and of different composition. Only in the case of $x_{\mathrm{Ar}}=0.1$ and the clusters with $N$ up to 931, the energy smoothly changes with temperature, and the inspection of snapshots showed that all krypton atoms form a commensurate patch while all argon atoms are located along the patch boundary [see figure 3 (a)].

In order to distinguish the commensurate and incommensurate atoms, we used the following order parameter [31, 37, 51]

$$
\phi(\mathbf{r})=\cos \left(\mathbf{q}_{1} \mathbf{r}\right)+\cos \left(\mathbf{q}_{2} \mathbf{r}\right)+\cos \left[\left(\mathbf{q}_{1}-\mathbf{q}_{2}\right) \mathbf{r}\right]
$$

and assumed that the atom is commensurate (incommensurate) when $\phi>0(\phi \leqslant 0)$.

In all other cases, the domain-wall structures were observed at sufficiently low temperatures. Figure 3 (b) shows the example of a snapshot recorded at $T=0.02$ and $x_{\mathrm{Ar}}=0.1$ for the larger patch made of 1333 atoms. It can be clearly seen that unlike in the smaller cluster of $N=931$ atoms, the cluster with $N=1333$ exhibits the domain-wall structure, and the walls are made nearly entirely of krypton atoms. The argon contributes to the walls only in the areas close to the patch boundaries. The patch boundaries 


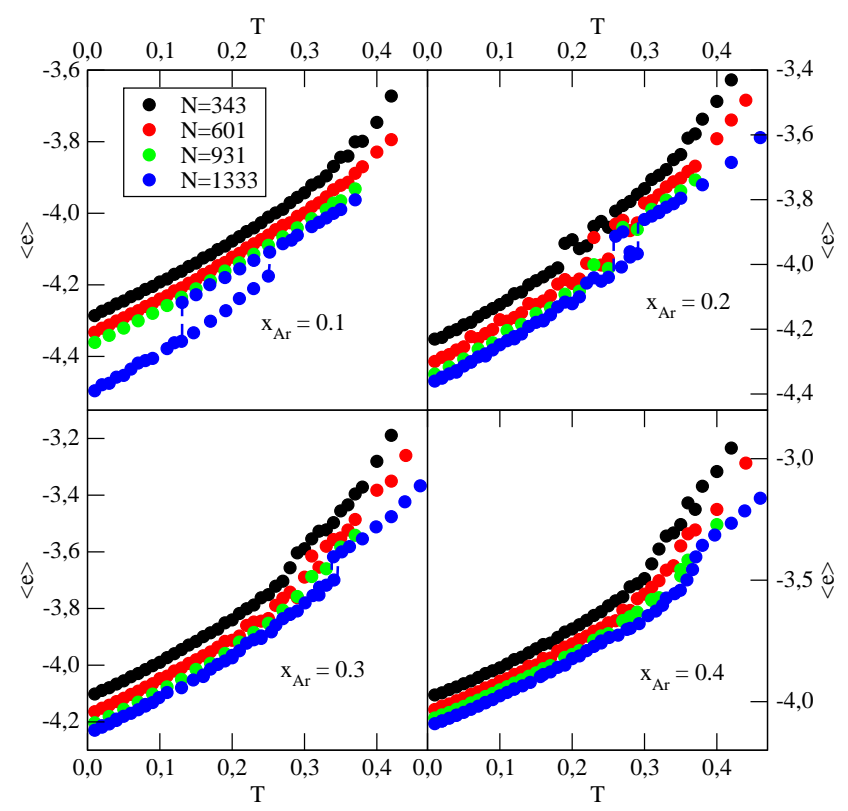

Figure 2. (Color online) The temperature changes of the potential energy (per particle) obtained for finite hexagonal patches of different size and of different argon mole fraction. The vertical blue lines mark the locations of transition between the mixed commensurate structure (stable at high temperatures) and the domain-wall structure (stable at low temperatures) in the largest clusters of $N=1333$ atoms, obtained from annealing and freezing runs.

are covered by a single layer of argon atoms. The structure of the IC phase is qualitatively similar to the IC phase in pure krypton monolayers of the density exceeding that of a fully filled C structure. However, the mechanism leading to the commensurate-incommensurate transition is quite different in both situations. In the case of a fully filled monolayer, the density excess over the value corresponding to a perfect $\mathrm{C}$ phase triggers the formation of domain walls. Upon the increase of density, the size of commensurate domains gradually decreases [30,65]. Ultimately, a dense IC phase without commensurate domains is formed close to the monolayer completion.

In finite mixed clusters the atoms close to the boundaries are strained and the magnitude of this strain depends on the number of argon atoms in the system. It is shown that the excess interfacial energy in pure krypton clusters is positive and it induces a force normal to the interface and pointing towards the patch interior. Evidently, this force is too weak to lead to the C-IC transition in the pure krypton clusters. The presence of a layer of argon atoms at the boundaries causes the interfacial energy to be considerably higher and hence induces a considerably larger force, which is sufficient to compress the patch and to trigger the C-IC transition. The number of argon atoms necessary to cover the entire patch boundary is proportional to the total length of the interface, which in turn is proportional to $N_{\mathrm{Kr}}^{1 / 2}$. For a small and fixed argon mole fraction in the system, the concentration of argon along the interface increases with the cluster size. Figure 3 (a) shows that in the case of $N=931$ and $x_{\mathrm{Ar}}=0.1$, argon atoms hardly cover the entire interface. On the other hand, when $N=1333$ [figure 3(b)], the entire interface is densely covered by argon and there is some excess of argon atoms which try to penetrate the patch and trigger the development of domain walls. When the argon concentration increases, the walls become mixed and for a large argon mole fraction are predominantly built of argon (see figure 4). We studied the changes of the wall composition resulting from the changes of argon concentration in the system for the clusters of different size with $N=601,931$ and 1333. We did not take into account the incommensurate atoms with less than 5 nearest neighbors. Such atoms are located along the patch boundaries, and the inspection of snapshots (see figure 3and 4) allowed us to assume that there is a single atomic layer of incommensurate atoms at the patch boundaries. Using such a procedure we were able to monitor the changes of the number of argon and krypton atoms incorporated within the walls and to estimate the argon concentration within the wall and along the patch boundaries. We performed a series of Monte Carlo simulations over a 

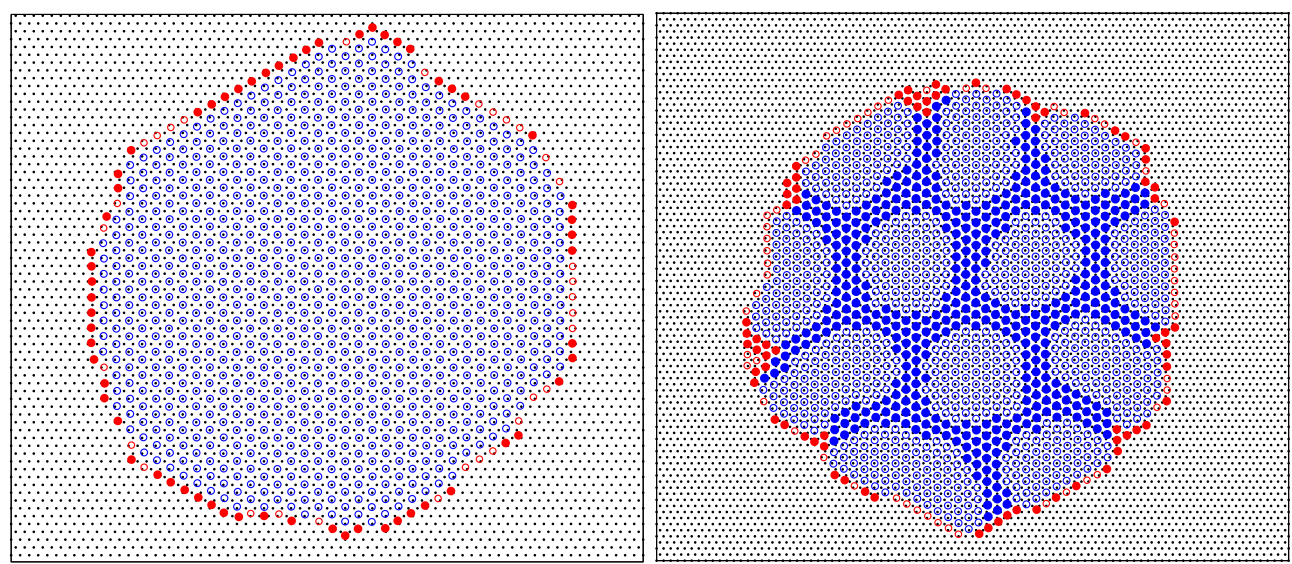

Figure 3. (Color online) The examples of configurations for the finite patches with $x_{\mathrm{Ar}}=0.1$ at $T=0.02$ consisting of 931 (part a) and 1333 (part b) atoms. Blue and red circles mark krypton and argon, respectively. The open circles correspond to the commensurate atoms with the order parameter $\phi\left(\mathbf{r}_{i}\right) \geqslant 0$, while the filled circles mark the incommensurate atoms with $\phi\left(\mathbf{r}_{i}\right)<0$.

certain temperature range between 0.01 and 0.3 for different cluster sizes and different argon mole fraction in the film. The examples of temperature changes of the wall composition are given in figure 5 (a), which shows the numbers of argon and krypton atoms forming the walls in the clusters of 1333 atoms and different argon mole fraction. The results demonstrate that for $x_{\mathrm{Ar}}=0.1$, the patch is commensurate at sufficiently high temperatures, and undergoes a transition to the incommensurate structure at $T=0.14 \pm 0.02$. The transition seems to be of the first order as suggested by rather large hysteresis along the freezing and annealing runs. In the case of $x_{\mathrm{Ar}}=0.2$, the transition occurs at higher temperature of about $T=0.20 \pm 0.02$ and is rounded. For the patches with a higher argon concentration, the incommensurate structure occurs over the entire range of temperatures studied. It is also evident that upon the increase of argon concentration in the film, the composition of walls changes from krypton rich to argon rich. Having the temperature changes of the wall composition we could estimate the ground state behavior of the systems studied.

The results are given in figure 5(b), which shows the changes of the argon mole fraction within the walls and at the patch boundaries versus the total argon mole fraction in the system. These results demonstrate that when the cluster size increases, the onset of the C-IC transition occurs at a lower argon concentration in the film. In the cluster with $N=601$, it occurs when $x_{\mathrm{Ar}} \approx 0.125$, in a larger cluster consisting of 931 atoms it starts when $x_{\mathrm{Ar}}$ is slightly higher than 0.1 , while in the largest cluster of $N=$

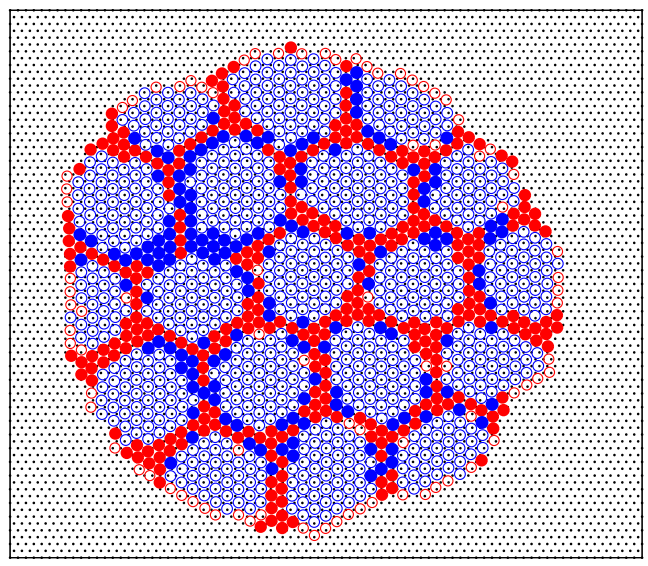

Figure 4. (Color online) The example of configuration for the cluster with $N=1333$ atoms and $x_{\mathrm{Ar}}=0.3$ at $T=0.06$. The labeling of atoms is the same as in figure 3 . 

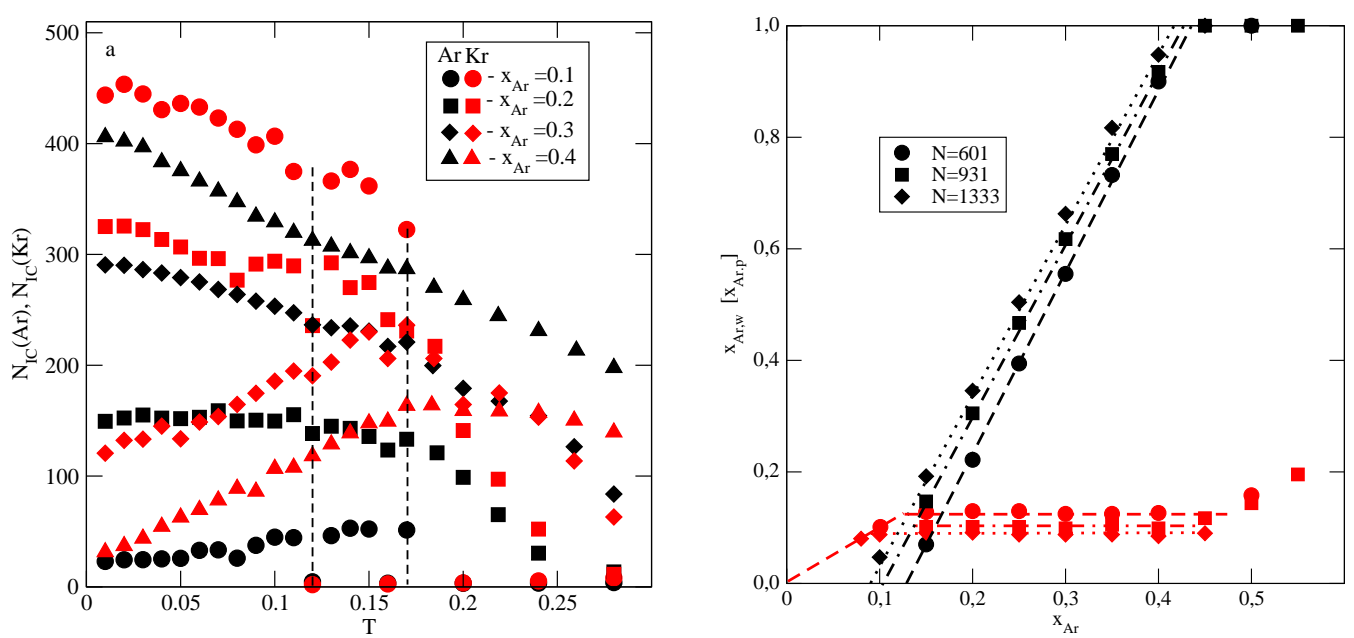

Figure 5. (Color online) (part a) The temperature changes of the numbers of argon and krypton atoms within the walls in finite clusters of $N=1333$ for the systems with different argon mole fraction equal 0.1 (circles), 0.2 (squares) 0.3 (diamonds) and 0.4 (triangles). Black and red symbols mark the numbers of argon and krypton atoms, respectively. (Part b). The mole fraction of argon within the wall (black symbols) and the mole fraction of argon atoms at the patch boundaries (red symbols) against the total mole fraction of argon atoms obtained for clusters of different total number of atoms (given in the figure). The lines serve only as a guidance.

1333 the transition is found already when $x_{\mathrm{Ar}} \approx 0.09$. Of course, when the argon mole fraction is lower than the limiting value necessary to trigger the C-IC transition, all argon atoms are located along the patch boundaries. The argon mole fraction within the walls increases linearly with the argon mole fraction in the film and for sufficiently large argon concentration the walls are entirely formed by argon. A further increase of the argon mole fraction leads to a gradual decrease of the size of krypton commensurate domains [51].

The inspection of configurations showed that when the walls are predominantly made of krypton atoms (at low argon concentrations) they are wider than the walls formed in the films with high argon concentration. The domain walls made of argon atoms only were observed to usually consist of just two rows of argon atoms and can be classified as heavy walls. The neighboring domains are shifted by the displacement vector of the length equal to $a_{\mathrm{gr}}$ [21]. The results obtained for different cluster sizes showed that the domains are rather small, and usually contain up to 10 commensurate krypton atoms along the line joining opposite walls. This is quite different from the already mentioned pure krypton IC phase, in which the domains are very large close to the C-IC transition and their size gradually decreases when the density becomes higher.

We also studied semi-infinite systems consisting of the rectangular commensurate domain of the size $L_{x} \times L_{y}$ with periodic boundary conditions applied along the $x$ and $y$-axes, but with free interfaces running along the $y$-axis. The simulation cell was of the size $L_{x}=60+\Delta$, with $\Delta=30$, and $L_{y}=30 \sqrt{3} / 2$.

Starting from a perfect $C$ structure, we estimated the ground state energies for three different configurations with the fixed numbers of $\mathrm{Ar}$ and $\mathrm{Kr}$ atoms equal to $N_{\mathrm{Ar}}=60$ and $N_{\mathrm{Kr}}=540$. The simulations were carried out at low temperatures, between 0.01 and 0.1 , using two types of algorithms, with and without the identity exchange attempts.

Three different systems were considered. In the first (i), two rows of krypton atoms adjacent to each boundary running along the $y$-axis were replaced by argon atoms. The second system (ii) contained only single rows of argon atoms at both free boundaries and the rest of Ar atoms formed a single wall consisting of two rows of atoms inside the $\mathrm{C}$ patch at a certain distance $r_{w}$ from one of the interfaces. The third system (iii) also consisted of single rows of argon atoms along the two free boundaries, but the rest of $\mathrm{Ar}$ atoms formed two walls, each made of one row of atoms located at the distance $r_{w}$ from the closer free interface. The calculations showed that in the systems (ii) and (iii) energy becomes independent of $r_{w}$ as soon as $r_{w}$ is larger than the assumed cutoff distance $r_{\max }$. The results given below were obtained for 
$r_{w}=10$.

The simulation without identity exchange attempts demonstrated that the system (ii) has a lower ground state energy (per particle) $\left(e_{\mathrm{i}} \approx-4.450\right)$ than the systems (i) $\left(e_{\mathrm{ii}} \approx-4.425\right)$ and (iii) $\left(e_{\mathrm{iii}} \approx-4.398\right)$. Moreover, the entire krypton patch in the system (i) was observed to form the $\mathrm{C}$ structure at low temperatures, while the vast majority of argon atoms were displaced to incommensurate positions due to a certain contraction resulting from the presence of free interfaces. In the case of system (ii), argon atoms were observed to form an incommensurate wall with the displacement vector between the commensurate domains at both sides of the wall corresponding to a heavy wall. The structure of system (iii) was found to be commensurate, just the same as in the case of system (i) Another Monte Carlo run, in which the identity exchange attempted was taken into account, performed for the system (i) demonstrated that already at very low temperatures the system structure spontaneously changes to that corresponding to the system (ii). Moreover, the two single rows of argon atoms inside the patch of the system (iii) were observed to merge into a single heavy wall consisting of two rows of argon atoms. We also performed a run using the algorithm involving the identity exchange attempts and the staring configuration with three rows of argon atoms at each free boundary. In this case, we observed the formation of two heavy walls running along the y-axis and made of two rows of argon atoms again.

We calculated the ground state energies of perfectly commensurate $\left(E_{\mathrm{c}, k}\right)$ and relaxed $\left(E_{\mathrm{rel}, k}\right)$ structures for the systems $k=(\mathrm{i})$, (ii) and (iii) as well as the energy gain (per the unit length of free interfaces) due to the relaxation

$$
e_{\mathrm{int}, k}=\left(E_{\mathrm{rel}, k}-E_{\mathrm{C}, k}\right) / L_{\mathrm{int}} .
$$

The energies (per atom) of perfectly ordered commensurate structures are very similar and equal to $e_{\mathrm{C}, 1}=-4.3748, e_{\mathrm{C}, 2}=-4.3699$ and $e_{\mathrm{C}, 3}=-4.36707$. On the other hand, the energy gains due to relaxation are considerably different and equal to $e_{\mathrm{int}, 1} \approx-1.16, e_{\mathrm{int}, 2} \approx-1.85$ and $e_{\mathrm{int}, 3} \approx-0.76$.

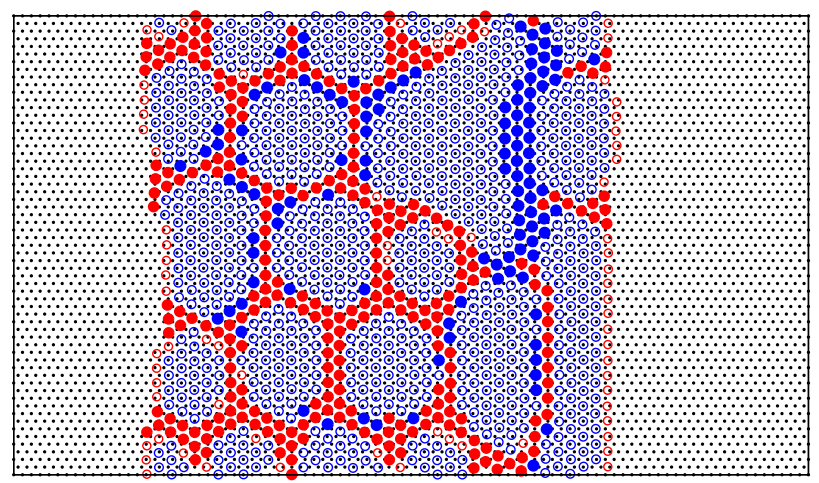

Figure 6. (Color online) The example of configuration for the rectangular slab of the size $60 \times 30 \sqrt{3}$ with $x_{\mathrm{Ar}}=0.2$ at $T=0.03$. Blue and red circles mark krypton and argon respectively. Circles with thin lines correspond to commensurate atoms with the order parameter $\phi\left(\mathbf{r}_{i}\right) \geqslant 0$, while circles with thick lines are for incommensurate atoms with $\phi\left(\mathbf{r}_{i}\right)<0$.

The above presented results demonstrate that the formation of heavy walls consisting of two rows of argon atoms inside the krypton patch leads to the most stable structure. It should be also noted that simulation for the systems with larger argon concentrations lead to the development of networks of heavy walls like that given in figure 6 rather than to a series of parallel walls running along the $y$-axis. This demonstrates that wall crossings contribute to the system stability and suggests that the wall crossing energy is negative. Of course, at finite temperatures the network of hexagonal walls is also stabilized by entropic effects, in particular, by the so-called breathing entropy [22, 23, 66].

Concluding this section, we should mention that from our earlier study [51] it follows that upon the increase of temperature the domain walls structure disappears and the mixed commensurate phase is formed at the temperatures below the melting point. 


\section{A simple one-dimensional Frenkel-Kontorova model}

In this section, we propose a simple modified one-dimensional Frenkel-Kontorova model [52, 53] that leads to qualitatively similar results as those discussed in the previous section.

Taking into account that the domain-wall structures that appear for submonolayer coverages are stable only at very low temperatures, we consider finite chains at zero temperature.

In general, the energy of the chain consisting of $N$ atoms of two different species $\mathrm{A}$ and B can be written as

$$
E=\frac{1}{2}\left\{\sum_{i=1}^{N-1} K_{i, i+1}\left[x_{i+1}-x_{i}-b_{i, i+1}\right]^{2}+\sum_{i=1}^{N} v_{i}\left[1-\cos \left(2 \pi x_{i} / a\right)\right]\right\} .
$$

In the above, we assumed that the interaction between a pair of nearest neighbors is harmonic and characterized by the force constant $K_{i, i+1}=K(\mathrm{~A}, \mathrm{~A}), K(\mathrm{~A}, \mathrm{~B})$ or $K(\mathrm{~B}, \mathrm{~B})$ and the equilibrium distance $b_{i, i+1}=b(\mathrm{~A}, \mathrm{~A}), b(\mathrm{~A}, \mathrm{~B})$ or $b(\mathrm{~B}, \mathrm{~B})$, depending on the composition of the pair. The second sum in equation (4.1) represents the contribution to the potential energy due to the periodic substrate field, with the distance between adjacent minima equal to $a$, and the amplitude of the external field $v_{i}$ is equal to $v_{\mathrm{A}}$ or $v_{\mathrm{B}}$.

In order to find the equilibrium configuration of such a mixed chain of the length $N$, one needs to specify the numbers of atoms A and B and their positions along the chain, and then to minimize the energy with respect to the set of $\left\{x_{i}\right\}$.

Here, we considered a few situations that are supposed to mimic the behavior of the previously discussed two-dimensional systems. We assumed that the component A is $\mathrm{Kr}$-like, while the component B is Ar-like. The equilibrium configuration of the chain made of atoms A only should correspond to the commensurate structure. On the other hand, the chain made of only B atoms should lead to an incommensurate floating structure. Assuming that the elastic constant $K(\mathrm{~A}, \mathrm{~A})$ is the unit of energy and the substrate lattice constant $a$ is the unit of length, we found out that the model with the parameters $v_{\mathrm{A}}=0.0055$ and $b(\mathrm{~A}, \mathrm{~A})=1.9$ describes reasonably well the commensurate structure. Note that by taking $b(\mathrm{~A}, \mathrm{~A})=1.9$ we assumed that in the commensurate structure every second potential well is occupied by an atom and that the misfit $m_{\mathrm{AA}}=b(\mathrm{~A}, \mathrm{~A})-2=-0.1$ is negative. This value of misfit is larger than in the case of $\mathrm{Kr} / \mathrm{Graphite}$ system, for which it is equal to about -0.06 [51], but assuming the value of the misfit equal to -0.06 , the FK model leads to the stable commensurate phase even for unreasonably low amplitudes of the surface field. To model Ar atoms we assumed that $v_{\mathrm{B}}=0.003, b(\mathrm{~B}, \mathrm{~B})=1.8$ and $K(\mathrm{~B}, \mathrm{~B})=0.9$ The parameters $K(\mathrm{~A}, \mathrm{~B})$ and $b(\mathrm{~A}, \mathrm{~B})$ are assumed to be given by the Lorentz-Berthelot mixing rules, so that

$$
K(\mathrm{~A}, \mathrm{~B})=\sqrt{K(\mathrm{~A}, \mathrm{~A}) K(\mathrm{~B}, \mathrm{~B})} \quad \text { and } \quad b(\mathrm{~A}, \mathrm{~B})=0.5[b(\mathrm{~A}, \mathrm{~A})+b(\mathrm{~B}, \mathrm{~B})] .
$$

We considered the chains that begin and terminate with atoms B. This assumption arises directly from the observation that in two-dimensional systems, the domain-wall structures appearing only after the peripheries of the cluster made of $\mathrm{Kr}$ atoms are covered with a filled single layer of Ar atoms. The situation in which argon atoms are located only at the ends of the chain corresponds to the model labeled as Model I. We considered two versions of this model assuming that there is only one argon atom at each chain end (Model I1) and that each end is decorated by two argon atoms (Model I2). Then, in the Model II, we assumed that at a certain position after the atom $N_{1}$, i.e., at the position $N_{1}+1$, a mixed wall of the thickness equal to $k$ atoms appears. The version of the model with a given value of $k$ is named Model $\operatorname{II}\left(k, N_{1}\right)$.

In order to consider mixed walls, we assumed that within the wall, the parameters representing the elastic constant, $K_{w}$, the equilibrium distance, $b_{w}$, and the amplitude of the substrate potential, $v_{w}$, are all dependent on the wall composition measured by the mole fraction of component $\mathrm{B}, x_{\mathrm{B}}$, and are given by:

$$
\begin{gathered}
K_{w}=x_{\mathrm{B}} K(\mathrm{~B}, \mathrm{~B})+\left(1-x_{\mathrm{B}}\right) K(\mathrm{~A}, \mathrm{~A}), \\
b_{w}=x_{\mathrm{B}} b(\mathrm{~B}, \mathrm{~B})=\left(1-x_{\mathrm{B}}\right) b(\mathrm{~A}, \mathrm{~A})
\end{gathered}
$$

and

$$
v_{w}=x_{\mathrm{B}} v_{\mathrm{B}}+\left(1-x_{\mathrm{B}}\right) v_{\mathrm{A}} .
$$


The pairs $\left(N_{1}, N_{1}+1\right)$ and $\left(N_{1}+k, N_{1}+k+1\right)$, i.e., at both sides of the wall, consist of one A atom and one wall atom, so that the respective elastic constants and equilibrium distances are given by

$$
K(\mathrm{~A}, w)=x_{\mathrm{B}} K(\mathrm{~A}, \mathrm{~B})+\left(1-x_{\mathrm{B}}\right) K(\mathrm{~A}, \mathrm{~A}) \quad \text { and } \quad b(\mathrm{~A}, w)=x_{\mathrm{B}} b(\mathrm{~A}, \mathrm{~B})=\left(1-x_{\mathrm{B}}\right) b(\mathrm{~A}, \mathrm{~A}) .
$$

The above assumption takes into account the fact that when the wall is made only of atoms $\mathrm{B}$, the interaction between the pairs $\left(N_{1}, N_{1}+1\right)$ and $\left(N_{1}+k, N_{1}+k+1\right)$ is the same as between the pairs $(\mathrm{A}, \mathrm{B})$ and $(N-1, N)$, which are equal to $K(\mathrm{~A}, \mathrm{~B})$ and $b(\mathrm{~A}, \mathrm{~B})$, respectively.

Of course, the above assumed additivity relations are too simple to properly describe the effects of wall composition on the behavior of adsorbed films, but we will demonstrate that even such a simple model leads to the results qualitatively very similar to those obtained for two-dimensional films.

Then, we introduced the displacements $u_{i}=x_{i} / b-2 i$, with $b$ being the equilibrium distance for the pair $i$ and $i+1$, and rewrote equation (4.1) in the form

$$
E=\frac{1}{2}\left\{\sum_{i=1}^{N-1} \hat{K}_{i, i+1}\left[u_{i+1}-u_{i}-m_{i, i+1}\right]^{2}+\sum_{i=1}^{N} \hat{v}_{i}\left[1-\cos \left(2 \pi u_{i}\right)\right]\right\},
$$

where the energy is expressed in units of $K(\mathrm{~A}, \mathrm{~A}) a^{2}$, and $\hat{K}_{i, i+1}=K_{i, i+1} /\left[K(\mathrm{~A}, \mathrm{~A}) a^{2}\right]$ and $\hat{v}_{i}=v_{i} /\left[K(\mathrm{~A}, \mathrm{~A}) a^{2}\right]$ and the misfits $m_{i, i+1}$ are defined as $m_{i, i+1}=b(\alpha, \beta) / a-2$ with $(\alpha, \beta)=(\mathrm{A}, \mathrm{A}),(\mathrm{A}, \mathrm{B}),(\mathrm{A}, w)$ or $(w, w)$.

In the case of Model I, the energy given by equation (4.7) depends on the number of argon atoms at the ends of the chain, while in the Models $\operatorname{II}\left(k, N_{1}\right)$ it depends on both the thickness of the wall $(k)$ and its position $\left(N_{1}\right)$.

One should note that the systems considered in the previous section were studied using Monte Carlo method allowing for the exchange of atoms identity, so that the distances between the patch boundary and the walls and the wall-wall distances inside the patch correspond to the equilibrium states. In the present one-dimensional model, the location of the wall is predetermined by $N_{1}$, and one needs to minimize the energy with respect to all displacements $\left\{u_{i}\right\}$ as well as with respect to $N_{1}$ in order to find the equilibrium state of the chain. On the other hand, the wall thickness and composition are controlled by the argon mole fraction in the chain. The first series of calculations is aimed at determining whether the assumed model predicts the formation of solitons when B atoms are located only at the chain boundaries, i.e., when $x_{\mathrm{B}}=0.0$ within the wall. The calculations carried out for different chain lengths, with $N$ ranging from 11 to 101, and using Model I1 always led to a single commensurate domain, i.e., to the same qualitative results as for pure A chain. The only effect of the B atoms located at the chain ends was an increase of atomic displacements near the chain ends (see figure 7). This result agrees very well with two-dimensional Monte Carlo simulations carried out for low $x_{\mathrm{Ar}}$, up to the value for which the

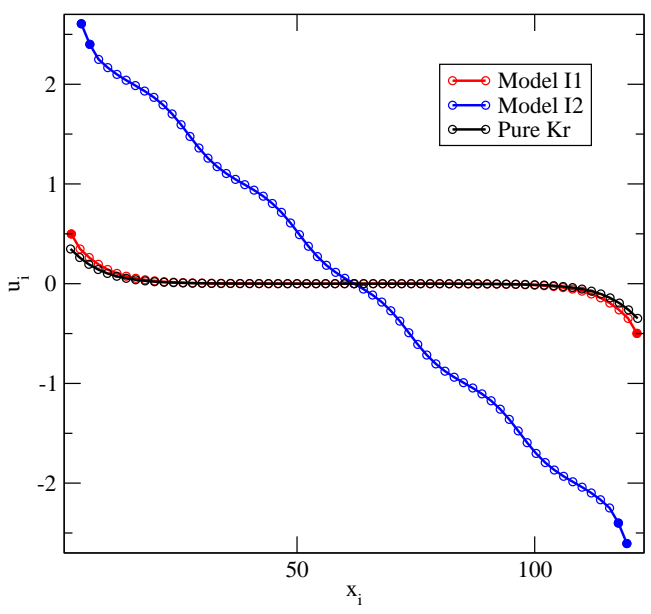

Figure 7. (Color online) Atomic displacements versus atomic positions for finite chains consisting of 41 atoms. Black circles and line correspond to pure chain of B atoms, while red and blue circles and lines correspond to the chains decorated by one and two atoms $\mathrm{A}$ at each end of the chain, respectively. 


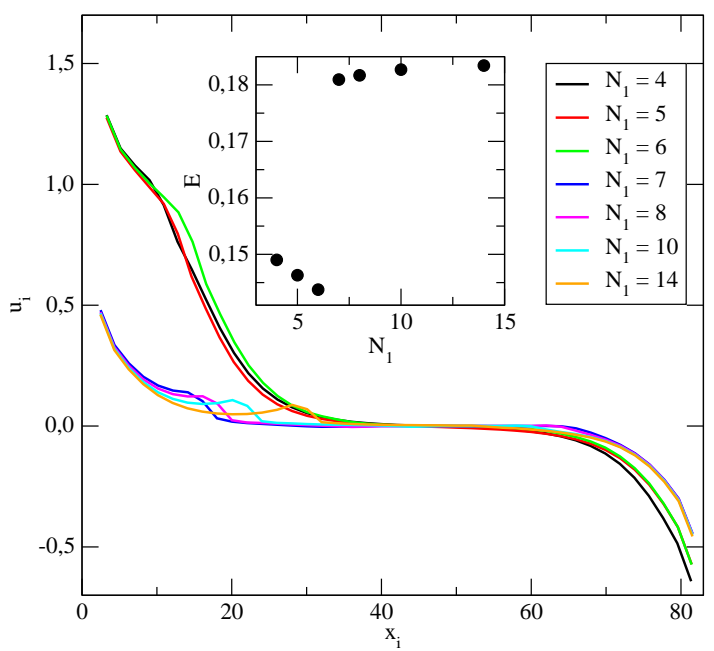

Figure 8. (Color online) The main figure shows atomic displacements versus atomic positions for finite chains consisting of 41 atoms with the ends decorated with one A atom and containing one A atom within the chain at the position $N_{1}+1$ (given in the legend and marked by a filled circle). The inset shows the changes of the system energy with the position of atom A within the chain.

entire boundary of commensurate $\mathrm{Kr}$ patch was covered by a single layer of Ar atoms. However, when the Model I2 was used, the introduced perturbation appeared to be sufficiently high to lead to the formation of solitons, i.e., the domain-wall structure (see figure 7). From the results of the previous section it follows that already at very low temperatures, the Monte Carlo simulation, starting with the configuration containing two rows of argon atoms at the boundaries, leads to the formation of a wall consisting of two rows of $\mathrm{Ar}$ atoms inside the $\mathrm{Kr}$ patch, and the boundaries are decorated by only a single layer of $\mathrm{Ar}$ atoms. Therefore, it is quite likely that the structure with two B atoms at each end of the chain is not the equilibrium state for the chain with four B atoms.

Then, we considered the systems described by Models $\operatorname{II}\left(k, N_{1}\right)$ with the wall of a thickness $k$ located inside the chain after the atom $N_{1}$. At first, we assumed that the chain length is fixed $(N=41)$ and studied the behavior of the model when the concentration of $B$ atoms within the wall changes.

This situation mimics finite submonolayer systems with the gradually increasing concentration of argon. In the two-dimensional systems, the number of atoms is proportional to $N^{2}$. Our choice of $N$ corresponds to clusters with the number of atoms equal to about $1500 \pm 100$ i.e., not much larger than those with 1333 atom that were studied by Monte Carlo simulations.

The calculations carried out within the Model II $\left(1, N_{1}\right)$ for the wall consisting entirely of argon $\left(x_{\mathrm{B}}=\right.$ 1.0) demonstrated that when $N_{1} \leqslant 6$, there develops a single soliton located behind the wall (see figure 8). The energy of the system acquires a minimum value when $N_{1}=6$ (see the inset a to figure 8 ).

When $N_{1}>6$, a single atom B inside the chain does not lead to the formation of a wall. Only a rather small perturbation appears close to the impurity atom (figure 8). Evidently, the commensurate stripe between the boundary and the atom $B$ is already wide enough to sustain the stress induced by the impurity.

In the case of the Model II $\left(2, N_{1}\right)$ with $N=41$, we found out that for $x_{\mathrm{B}}$ up to 0.04 , the commensurate structure is a stable state for all possible values of $N_{1}$. However, already for $x_{\mathrm{B}}=0.05$, the system undergoes a transition to the domain-wall structure. Figure 9 shows the atomic displacements obtained for a series of systems with $x_{\mathrm{B}}$ between 0.05 and 1.0. Thus, the results indicate that even when the perturbation introduced by a small concentration of component B within the wall is weak, it leads to the development of solitons. Two types of behavior can be singled out. For $x_{B} \in(0.05,0.6)$, there are three solitons and with the increase of $x_{\mathrm{B}}$, the $\mathrm{B}$ atoms gradually approach the position of the first soliton. In the second regime, only two solitons appear and the B atoms are located right within the wall.

Of course, the results obtained for a high concentration of atoms B in the wall, do not correspond well to the 2D systems studied using Monte Carlo simulations. For a large concentration of atoms B, one would expect the formation of several mixed walls with lower values of $x_{\mathrm{B}}$ rather than the appearance 


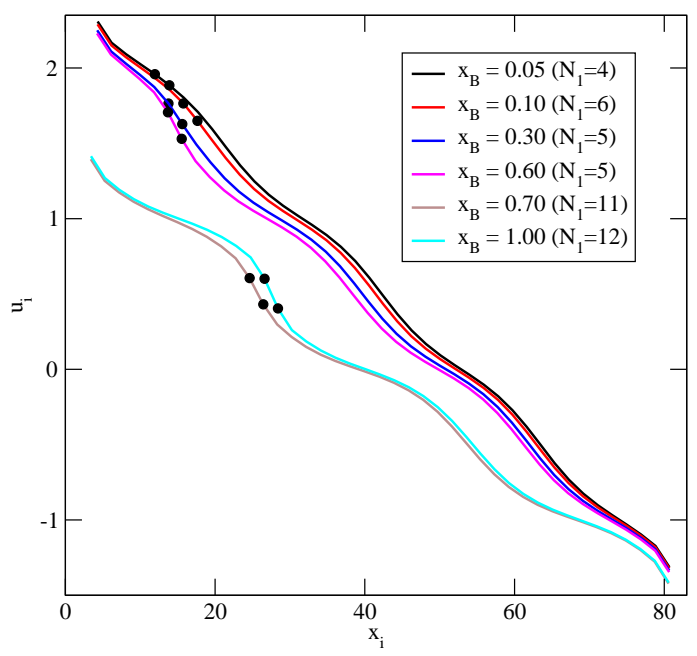

Figure 9. (Color online) Atomic displacements versus atomic positions for finite chains consisting of 41 atoms with the ends decorated with one A atom and containing two A atoms within the chain at the positions marked by black circles obtained for different compositions of the wall inside the chain and corresponding to the location of the wall that minimizes the system energy.

of a single wall with a high value of $x_{\mathrm{B}}$. Nevertheless, the model presented catches the most important features of the C-IC transition in finite systems driven by the presence of impurities.

\section{Summary}

Using Monte Carlo simulation method we studied the C-IC transition which occurs at low temperatures in finite clusters of $\mathrm{Kr}$ atoms contaminated with argon. It is demonstrated that pure krypton clusters form only the commensurate structure. This is consistent with experimental data clearly demonstrating that the commensurate structure is stable as long as the density does not exceed the density of a fully covered commensurate phase [41]. Also, the clusters retain a commensurate structure when small amounts of argon are added to the system. It is established that argon atoms are predominantly located along the cluster boundaries and the $\mathrm{C}$ structure is stable up to the point when the entire cluster boundary is covered with a single layer of argon atoms. As soon as the argon concentration becomes higher than this threshold value, the system undergoes the C-IC transition leading to the formation of the domain-wall structure. The walls separating the commensurate domains are found to be heavy and their composition to change with the argon concentration. In particular, we found out that the argon concentration within the wall changes linearly with the total argon mole fraction in the system. Thus, at a certain upper limit of the argon concentration in the film, the walls are made of argon only, while the cluster boundaries are still covered with a single atomic layer of argon. A further increase of argon concentration causes the walls made of argon and the argon layer along the patch boundaries to become thicker. It should be noted that the size of commensurate domains does not depend much upon the argon concentration and the domains are made of pure krypton. This demonstrates that the mixing does not occur in the $\mathrm{C}$ phase. On the other hand, the mixing does occur within the incommensurate walls over a rather wide range of argon concentration.

The above described behavior can be attributed to the rather large changes in the interaction energies between different pairs ( $\mathrm{Kr}-\mathrm{Kr}, \mathrm{Ar}-\mathrm{Kr}$ and Ar-Ar) when the system undergoes the C-IC transition. In the $\mathrm{C}$ phase, the distance between neighboring atoms is determined by the structure of the graphite lattice. A considerably smaller misfit of krypton compared to argon, causes the system to maximize the number of $\mathrm{Kr}-\mathrm{Kr}$ pairs and to avoid the appearance of $\mathrm{Ar}-\mathrm{Kr}$ and $\mathrm{Ar}-\mathrm{Ar}$ pairs. In the incommensurate state, i.e., within the walls, the energy of interaction between Ar-Kr and Ar-Ar pairs is considerably higher since the distances between nearest neighbors within the heavy walls are considerably lower and closer to the locations of the LJ potential minima. This effect enhances the tendency toward mixing. Besides, the 
stability of walls is further enhanced by rather large entropic effects.

We also showed that similar C-IC transition emerges from a simple one-dimensional FrenkelKontorowa model applied to finite mixed chains. It is demonstrated that for appropriately chosen parameters of the model, its behavior is qualitatively very similar to that obtained from Monte Carlo simulations of two-dimensional systems.

\section{References}

1. Jaubert M., Glachant A., Bienfait M., Boato G., Phys. Rev. Lett., 1981, 46, 1679; doi 10.1103/PhysRevLett.46.1679

2. Krim J., Suzanne J., Shechter H., Wang R., Taub H., Surf. Sci., 1985, 162, 446; doi 10.1016/0039-6028(85)90933-1

3. Fain S.C., Chinn M. D., Diehl R.D., Phys. Rev. B, 1980, 21, 4170; doi 10.1103/PhysRevB.21.4170

4. Stephens P.W., Heiney P.A., Birgeneau R.J., Horn P.M., Moncton D.E., Brown G.S., Phys. Rev. B, 1984, 29, 3512; doi $10.1103 /$ PhysRevB.29.3512

5. Stephens P.W., Heiney P.A., Birgeneau R.J., Horn P.M., Phys. Rev. Lett., 1979, 43, 47; doi 10.1103/PhysRevLett.43.47

6. Imbihl R., Behm R.J., Christmann K., Ertl G., Matsushima T., Surf. Sci., 1982, 117, 257; doi 10.1016/0039-6028(82)90506-4.

7. Grunze M., Kleban P.H., Unertl W.N., Rys F.S., Phys. Rev. Lett., 1983, 51, 582; doi 10.1103/PhysRevLett.51.582

8. Usachov D., Dobrotvorskii A.M., Varykhalov A., Rader O., Phys. Rev. B, 2008, 78, 085403; doi 10.1103/PhysRevB.78.085403

9. Faisal A.Q.D., Hamini M., Raynerd G., Venables J.A., Phys. Rev. B, 1986, 34, 7440; doi 10.1103/PhysRevB.34.7440

10. Laetherman G.S., Diehl R.D., Phys. Rev. B, 1996, 53, 4939; doi 10.1103/PhysRevB.53.4939

11. Park C., Bauer E., Kramer H.M., Surf. Sci., 1982, 119, 251; doi 10.1016/0039-6028(82)90295-3

12. Glachant A., Jaubert M., Bienfait M., Surf. Sci., 1982, 115, 219; doi 10.1016/0039-6028(82)90670-7

13. Kern K., David R., Palmer R.L., Comsa G., Phys. Rev. Lett., 1986, 56, 620; doi 10.1103/PhysRevLett.56.620

14. Freimuth H., Wichert H., Surf. Sci., 1987, 189-190, 548; doi 10.1016/S0039-6028(87)80480-6

15. Andryushechkin B.V., Eltsov K.N., Shevlyuga V.M. Surf. Sci., 2000, 470, L63; doi 10.1016/S0039-6028(00)00896-7

16. Villain J., In: Ordering in Strongly fluctuating Condensed Matter Systems, Riste T. (Ed.), Plenum Press, New York, 1980, 221-260.

17. Bak P., Rep. Prog. Phys., 1982, 45, 587; doi 10.1088/0034-4885/45/6/001

18. Gordon M.B., Villain J., J. Phys. C: Solid State Phys., 1985, 18, 3919; doi 10.1088/0022-3719/18/20/018

19. Gordon M.B., Lançon F., J. Phys. C: Solid State Phys., 1985, 18, 3929; doi 10.1088/0022-3719/18/20/019

20. Pokrovsky V.L., Talapov A.L., Theory of Incommensurate Crystals, Soviet Scientic Reviews Supplement Series, Physics, Vol. 1, Harwood, New York, 1984.

21. Huse D.A., Fisher M., Phys. Rev. B, 1984, 29, 239; doi 10.1103/PhysRevB.29.239

22. Villain J., Gordon M.B., Surf. Sci., 1983, 125, 1; doi 10.1016/0039-6028(83)90443-0

23. Den Nijs M., In: Phase Transitions and Critical Phenomena, Vol. 12, Domb C., Lebowitz J.L. (Eds.), Acedemic Press, London, 1988, 220-333.

24. Gottlieb J.M., Bruch L.W., Phys. Rev. B, 1991, 44, 5750; doi 10.1103/PhysRevB.44.5750

25. Schulz J.H., Phys. Rev. B, 1980, 22, 5274; doi 10.1103/PhysRevB.22.5274

26. Bak P., Mukamel P., Villain J., Wentowska K., Phys. Rev. B, 1979, 19, 1610; doi 10.1103/PhysRevB.19.1610

27. Coppersmith S.N., Fisher D.S., Halperin B.I., Lee P.A., Brinkman W.F., Phys. Rev. B, 1982, 25, 349; doi 10.1103/PhysRevB.25.349

28. Saito Y., Phys. Rev. B, 1981, 24, 6652; doi 10.1103/PhysRevB.24.6652

29. Abraham F.F., Koch S.W., Rudge W.E., Phys. Rev. Lett., 1982, 49, 1830; doi 10.1103/PhysRevLett.49.1830

30. Schöbinger M., Abraham F.F., Phys. Rev. B, 1985, 31, 4590; doi 10.1103/PhysRevB.31.4590

31. Kim H.-Y., Steele W.A., Phys. Rev B, 1992, 45, 6226; doi 10.1103/PhysRevB.45.6226

32. Sokoloff J.B., Phys. Rev. B, 1980, 22, 2506; doi 10.1103/PhysRevB.22.2506

33. Houlrik J.M., Landau D.P., Phys. Rev. B, 1991, 44, 8962; doi 10.1103/PhysRevB.44.8962

34. Patrykiejew A., Sokołowski S., Zientarski T., Binder K., Surf. Sci., 1999, 421, 308; doi 10.1016/S0039-6028(98)00858-9

35. Patrykiejew A., Sokołowski S., Binder K., Surf. Sci., 2002, 512, 1; doi 10.1016/S0039-6028(02)01702-8

36. Parlinski K., Chapuis G., Phys. Rev. B, 1994, 49, 11643; doi 10.1103/PhysRevB.49.11643

37. Houlrik J.M., Landau D.P., Knak Jensen S.J., Phys. Rev. E, 1994, 50, 2007; doi 10.1103/PhysRevE.50.2007

38. Stephens P.W., Heiney P.A., Birgeneau R.J., Horn P.M., Moncton D.E., Brown G.S., Phys. Rev. B, 1984, 29, 3512; doi 10.1103/PhysRevB.29.3512

39. Specht E.D., Mak A., Peters C., Sutton M., Birgeneau R.J., D’Amico K.L., Moncton D.E., Nagler S.E., Horn P.M., Z. Phys. B: Condens. Matter, 1987, 69, 347; doi 10.1007/BF01307294 
40. Bruch L.W., Cole M.W., Zaremba E., Physical Adsorption: Forces and Phenomena, Clarendon Press, Oxford, 1997.

41. Bruch L.W., Diehl R.D., Venables J.A., Rev. Mod. Phys., 2007, 79, 1381; doi 10.1103/RevModPhys.79.1381.

42. Butler D.M., Litzinger J.A., Stewart G.A., Griffiths R.B., Phys. Rev. Lett., 1979, 42, 1289; doi 10.1103/PhysRevLett.42.1289.

43. Gooding R.J., Joos B., Bergersen B., Phys. Rev. B, 1983, 27, 7669; doi 10.1103/PhysRevB.27.7669

44. Nielsen M., Als-Nielsen J., Bohr J., McTague J.P., Moncton D.E., Stephens P.W., Phys. Rev. B, 1987, 35, 1419; doi 10.1103/PhysRevB.35.1419

45. Stephens P.W., Goldman A.I., Heiney P.A., Bancel P.A., Phys. Rev. B, 1986, 33, 655; doi 10.1103/PhysRevB.33.655

46. Bohr J., Nielsen M., McTague J.P., Als-Nielsen J., Kjaer K., Surf. Sci., 1983, 125, 181; doi $10.1016 / 0039-6028(83) 90451-X$

47. De Beauvais C., PhD Thesis, Université Paris 7, 1986.

48. Ma J., Carter E.D., Kleinberg H.B., Phys. Rev. B, 1998, 57, 9270; doi 10.1103/PhysRevB.57.9270

49. Singleton J.H., Halsey G.D., J. Phys. Chem., 1954, 58, 1011; doi 10.1021/j150521a019

50. Roth M.W., Langmuir, 1999, 15, 2169; doi 10.1021/la980171610.1021

51. Patrykiejew A., Rżysko W., Sokołowski S., J. Phys. Chem. C, 2012, 112, 753; doi 10.1021/jp208323b

52. Patrykiejew A., Sokołowski S., Condens. Matter Phys., 2014, 17, 23605; doi 10.5488/CMP.17.23605

53. Frenkel Y.I., Kontorova T., Zh. Eksp. Theor. Fiz., 1938, 8, 1340 (in Russian).

54. Lomdahl P.S., Srolovitz D.J., Phys. Rev. Lett., 1986, 57, 2702; doi 10.1103/PhysRevLett.57.2702

55. Hamilton J.C., Phys. Rev. Lett., 2002, 88, 126101; doi 10.1103/PhysRevLett.88.126101

56. Daruka I., Hamilton J.C., J. Phys.: Condens. Matter, 2003, 15, 1827; doi 10.1088/0953-8984/15/12/302

57. Kawaguchi T., Matsukawa H., Phys. Rev. B, 1997, 56, 4261; doi 10.1103/PhysRevB.56.4261.

58. Braun O.M., Kivshar J.S., Phys. Rep., 1998, 306, 1; doi 10.1016/S0370-1573(98)00029-5.

59. Sharma S.R., Bergersen B., Joos B., Phys. Rev. B, 1984, 29, 6335; doi 10.1103/PhysRevB.29.6335

60. Braiman Y., Baumgarten J., Jortner J., Klafter J., Phys. Rev. Lett., 1990, 65, 2398; doi 10.1103/PhysRevLett.65.2398

61. Braiman Y., Baumgarten J., Klafter J., Phys. Rev. B, 1993, 47, 11159; doi 10.1103/PhysRevB.47.11159

62. Vanossi A., Franchini A., Bortolani V., Surf. Sci., 2002, 502-503, 437; doi 10.1016/S0039-6028(01)01990-2

63. Mikheikin I.D., Sakun V.P., Kuznetsov M.Yu., Makhonina E.V., Pervov V.S., J. Mater. Sci., 2007, 42, 1122; doi 10.1007/s10853-006-1455-z

64. Landau D.P., Binder K., A Guide to Monte Carlo Simulation in Statistical Physics, Cambridge University Press, Cambridge, 2000.

65. Abraham F.F., Rudge W.E., Auerbach D.J., Koch S.W., Phys. Rev. Lett., 1984, 52, 445; doi 10.1103/PhysRevLett.52.445.

66. Villain J., Surf. Sci., 1980, 97, 219; doi 10.1016/0039-6028(80)90115-6

\title{
Механізм формування структури доменної стінки в субмоношарових плівках Ar-Kr на графіті
}

\author{
А. Патрикієв, С. Соколовський \\ Відділ моделювання фізико-хімічних процесів, хімічний факультет, Університет імені Марії \\ Склодовської-Кюрі, 20031 Люблін, Польща
}

\begin{abstract}
Використовуючи метод моделювання Монте-Карло в канонічному ансамблі, ми дослідили перехід від співвимірної до неспіввимірної фази у двовимірних скінчених змішаних кластерах $\mathrm{Ar}$ i Kr, адсорбованих на графітній базисній площині при низьких температурах. Показано, що перехід відбувається, коли концентрація аргону перевищує значення, необхідні для покриття периферій кластера. Неспіввимірна фаза демонструє подібну структуру доменної стінки до тої, яка була спостережена у плівках чистого криптону при густинах, що перевищують ідеальну $(\sqrt{3} \times \sqrt{3}) R 30^{\circ}$ співвимірну фазу, але розмір співвимірних доменів значно не змінюється зі зміною розміру кластера. Коли концентрація аргону зростає, склад доменних стінок змінюється, в той час як співвимірні домени складаються з чистого криптону. Ми побудували просту одновимірну модель Френкеля-Конторової, яка приводить до результатів, що добре якісно узгоджуються з результатами Монте-Карло, отриманими для двовимірних систем.
\end{abstract}

Ключові слова: адсорбція сумішей, переходи від співвимірної до неспіввимірної фази, моделювання Монте Карло, скінчені системи, модель Френкеля-Конторової 